\title{
Le système éducatif birman : transformations en cours et enjeux d'avenir
}

\section{Kam Tung Tuang Suante}

Traducteur : Sylvaine Herold

\section{OpenEdition Journals}

Édition électronique

URL : https://journals.openedition.org/ries/8102

DOI : $10.4000 /$ ries.8102

ISSN : 2261-4265

Éditeur

France Education international

Édition imprimée

Date de publication : 1 avril 2019

Pagination : 31-38

ISBN : 978-2-85420-623-4

ISSN : $1254-4590$

\section{Référence électronique}

Kam Tung Tuang Suante, «Le système éducatif birman : transformations en cours et enjeux d'avenir », Revue internationale d'éducation de Sèvres [En ligne], 80 | avril 2019, mis en ligne le 01 avril 2021, consulté le 24 juin 2021. URL : http://journals.openedition.org/ries/8102 ; DOI : https://doi.org/ $10.4000 /$ ries.8102

Ce document a été généré automatiquement le 24 juin 2021.

(c) Tous droits réservés 


\title{
Le système éducatif birman : transformations en cours et enjeux d'avenir
}

\author{
Kam Tung Tuang Suante \\ Traduction : Sylvaine Herold
}

\section{Une longue histoire éducative}

1 Le Myanmar (Birmanie), pays multiethnique à faible revenu, est divisé en sept États sur la base des sept minorités ethniques du pays - les Chins, les Kachins, les Karens, les Kayahs, les Môns, les Arakanais (Rakhine) et les Shans -, sept régions et un territoire de l'Union. Le groupe ethnique birman représente environ $68 \%$ de la population. D'après le recensement de 2014, la Birmanie compte 52 millions d'habitants et $89,8 \%$ de la population pratique le bouddhisme (CSO, 2017).

2 Les monastères bouddhistes ont une longue tradition d'enseignement des textes religieux et d'alphabétisation de base dans les régions birmanes et dans l'État môn. La scolarisation a commencé à se généraliser en Birmanie après l'arrivée des missionnaires chrétiens, au début du XIX siècle et pendant l'administration britannique (1824-1942, 1945-1948). Un système éducatif centralisé a été adopté lorsque la Birmanie est devenue un État indépendant en 1948. Le coup d'État militaire de 1964, puis la dictature à parti unique, de 1974 à 1988, ont limité l'enseignement privé et fait de l'enseignement public un outil de propagation de la dictature et de «birmanisation ». Ce terme désigne l'imposition tactique de l'identité, de la langue, de l'histoire et de la culture birmanes, mais également de la religion dominante, le bouddhisme, aux minorités ethniques et religieuses, notamment par le biais de la scolarisation (South et Lall, 2016). En retour, les minorités ethniques ont développé des systèmes scolaires dans leurs zones de contrôle, pour résister au processus de birmanisation, conserver et régénérer leur identité, leur histoire, leur culture et leur langue (ibid.). 
3 Environ 14,91 millions d'enfants âgés de 5 à 19 ans étaient scolarisés en 2014 (CSO, 2017) et 8,9 millions étaient inscrits dans l'éducation de base au cours de l'année scolaire 2016-2017 (CSO, 2017). 225178 étudiants à temps plein et 411164 étudiants à distance étaient inscrits dans l'enseignement supérieur en 2015 (NESP, 2016). 270909 élèves fréquentaient les écoles monastiques bouddhistes en 2016-2017 (CSO, 2017) et Jollife (2014) a estimé que plus de 227969 enfants seraient scolarisés dans des écoles ethniques et des camps de réfugiés. La junte militaire au pouvoir de 1988 à $2011 \mathrm{a}$ développé la scolarisation dans les zones frontalières à partir de 1999; quelque 6719 enfants y fréquentaient des établissements de formation en 2017-2018 (MOBA, 2019).

\section{Des changements importants depuis 2011}

4 Des changements éducatifs importants ont été mis en œuvre après la transition démocratique de 2011. La Birmanie affiche la volonté de devenir un pays à revenu intermédiaire d'ici 2030 et l'éducation est considérée comme le fondement du développement économique, de l'établissement d'une paix durable et de la transformation sociale du pays.

5 Ont ainsi été construits 1838 écoles primaires, 390 collèges et 629 lycées entre 2012 et 2017. Les ratios élèves/enseignant sont demeurés stables : $23 / 1$ dans le primaire, 29/1 dans le secondaire inférieur et 23/1 dans le secondaire supérieur en 2016-2017.

6 Depuis 2011, l'enseignement privé est autorisé, qui comptait 110101 élèves en 2016-2017 (CSO, 2017). En 2012, le gouvernement a conduit un examen global du secteur éducatif, à partir duquel il a promulgué la loi sur l'éducation nationale en 2014 (National Education Law, NEL), voté un amendement en 2015 et lancé le Plan stratégique national pour l'éducation (National Education Strategic Plan, NESP), en 2016. La loi sur l'éducation nationale, qui prévoit de porter les dépenses éducatives à $20 \%$ des dépenses totales du budget, a défini neuf objectifs éducatifs en mettant l'accent sur le développement intégral des enfants, les droits de l'Homme, l'esprit d'union et la préservation de la culture, le développement économique et la qualité de vie. Cependant, la portée du droit à l'éducation se limite aux citoyens birmans et demeure controversé, s'agissant des millions de descendants de Chinois, d'Indiens et d'autres nationalités n'ayant pas encore obtenu la pleine citoyenneté en Birmanie.

\section{Les transformations de l'éducation formelle de base}

7 L'éducation formelle comprend la maternelle, l'éducation de base, l'enseignement technique et professionnel et l'enseignement supérieur. Le Plan stratégique national prévoit deux phases et neuf changements transformationnels. La première phase (NESP, 2016-2021) consiste à mettre en œuvre les réformes fondamentales nécessaires pour atteindre l'objectif d'« amélioration de l'enseignement et de l'apprentissage, de l'enseignement et de la formation professionnels, de la recherche et de l'innovation conduisant à des progrès mesurables des résultats des élèves dans l'ensemble des écoles et des établissements d'enseignement ».

8 La loi sur l'éducation considère le niveau maternel, où les enfants sont admis à l'âge de 5 ans, comme le fondement de l'éducation de base. Le Plan stratégique national prévoit 
d'augmenter le taux d'inscription en maternelle, de créer de nouvelles classes, d'intégrer davantage les parents dans les activités de l'école et de mettre en place un programme national de formation des enseignants de maternelle. Il soutient et subventionne également les classes de maternelle gérées par d'autres ministères et organisations de la société. Il vise en outre à élaborer des normes minimales pour l'éducation maternelle et à collecter des données de référence de recensement et de cartographie préscolaires. Cependant, la qualité et l'inclusivité de l'éducation maternelle demeurent un défi, car la formation des enseignants de la petite enfance et l'éducation inclusive ne sont pas bien mises en œuvre. Exclusivement théorique, la formation continue des enseignants de maternelle est en outre inefficace.

9 La structure scolaire comprend désormais douze années de scolarité après la maternelle - cinq années de primaire, quatre années de secondaire inférieur et trois années de secondaire supérieur. Afin d'augmenter le taux de scolarisation et de réduire le taux d'abandon scolaire, le gouvernement a supprimé les frais de scolarité jusqu'au secondaire en 2015-2016 et il fournit également gratuitement les manuels et uniformes aux élèves. Il a supprimé les frais d'inscription, ceux liés aux fournitures scolaires et aux associations parents-enseignants, accorde des bourses aux élèves défavorisés et subventionne les établissements scolaires pour les travaux d'entretien.

\section{Un nouveau curriculum}

10 La nouvelle structure scolaire et le curriculum révisé ont été introduits au cours de l'année scolaire 2016-2017. Le nouveau curriculum fait du birman, de l'anglais et des mathématiques des matières obligatoires et introduit l'éducation morale et civique, l'étude sociale, la préparation à la vie active, l'éducation physique et les arts. Le ministère de l'Éducation propose actuellement aux enseignants une formation courte et itinérante à ces programmes. Au premier cycle du secondaire, les élèves étudient l'histoire, la géographie et les sciences en plus des matières obligatoires. Au deuxième cycle du secondaire, les élèves peuvent choisir trois matières parmi l'histoire, la géographie, l'économie, la biologie, la physique, la chimie et une option birman additionnelle, en plus des matières obligatoires. Si la scolarité intègre les compétences pour la vie, les arts et l'éducation physique, ces matières ne sont pas prises en compte et les élèves ne leur accordent que peu d'importance.

\section{Le poids de la « birmanisation » sur les minorités ethniques}

11 Par ailleurs, les minorités ethniques considèrent que l'expansion de la scolarisation renforce la birmanisation de l'éducation, en l'absence de reconnaissance de la diversité et de contenus contextuels (South et Lall, 2016). S'il est permis d'apprendre les langues minoritaires en tant que matières à l'école primaire et de les utiliser en complément de la langue d'enseignement birmane, il n'est pas permis d'utiliser des langues minoritaires comme seule langue d'enseignement et de développement du curriculum. Bien que la loi sur l'éducation s'attache à promouvoir et à préserver les différentes cultures, arts, langues et littératures de la Birmanie, les contenus et les concepts des nouveaux programmes continuent d'être dominés par le contexte birman et le bouddhisme. Par exemple, parmi les six dirigeants historiques présentés dans les 
nouveaux manuels d'étude sociale comme " personnalités historiques dont je suis fier " figurent quatre rois birmans, un héros birman et une reine de l'ethnie Môn. Bien que la loi sur l'éducation affirme que « les établissements scolaires doivent être exempts de toute ingérence religieuse ou politique », les dogmes du bouddhisme sont fréquemment intégrés au curriculum. Alors que la constitution nationale proclame la République de l'Union de Birmanie, le programme présente à maintes reprises le pays - l'Union de Birmanie - comme la succession de royaumes birmans, le bouddhisme comme la religion du pays et l'ethnie birmane comme son groupe ethnique idéal, en contradiction apparente avec les principes de la démocratie et de la république.

\section{L'évaluation de l'amélioration des résultats scolaires}

12 Le Plan stratégique national entend atteindre des progrès scolaires mesurables à l'horizon 2020-2021 et considère les évaluations comme le moyen "pour obliger les établissements et les enseignants à rendre compte de leurs performances et pour élaborer et affiner les politiques publiques ».

Le département des examens du Myanmar (Department of Myanmar Examination, DME) est chargé de la réforme des évaluations et des examens. Les examens publics en fin de $5 \mathrm{e}$ et de $9 \mathrm{e}$ années ont été réintroduits en 2015. Les résultats à l'examen de fin d'études secondaires, en fin de $11^{\mathrm{e}}$ année, constituent toujours le principal critère d'admission dans l'enseignement supérieur. Le DME prévoit de modifier le format des questions lors de l'examen de fin d'études secondaires 2019. Il restera à déterminer si les modifications apportées au format des questions, et non au système d'examen en tant que tel, permettent de mesurer les résultats attendus dans le nouveau curriculum - les compétences pour le XXI ${ }^{e}$ siècle.

\section{La formation et le développement professionnel des enseignants}

La formation des enseignants est dispensée par 25 instituts pédagogiques (education colleges), deux facultés d'éducation et une université en faveur du développement des ethnies nationales de l'Union. Les instituts pédagogiques proposent une formation d'un an aux enseignants du primaire et de deux ans aux enseignants du secondaire inférieur. Depuis 2014, la formation des enseignants du secondaire supérieur, jusqu'alors de quatre ans, est passée à cinq ans dans les trois universités. Le gouvernement verse une allocation mensuelle de 30000 kyats $^{1}$ par stagiaire et propose un cours d'enrichissement en anglais pour les formateurs d'enseignants, avec l'aide du British Council. Le gouvernement a recruté un plus grand nombre de tuteurs pédagogiques et les 25 instituts (dont quatre ont été ouverts après 2014) deviendront des instituts de formation pédagogique diplômants en quatre ans, en décembre 2019. Le Plan stratégique national prévoit de mettre en œuvre des programmes de formation en matière de langues minoritaires et d'enseignement inclusif pour les enseignants et de renforcer leur développement professionnel continu. Le nombre d'enseignants a augmenté entre 2012-2013 et 2016-2017 pour atteindre 41614 enseignants de primaire, 31554 enseignants de secondaire inférieur et 12278 enseignants de secondaire supérieur. Le gouvernement décide également désormais de la nomination d'un 
nombre prévisionnel de personnel non enseignant pour chaque niveau scolaire (par exemple, agents d'entretien, employés de bureau, agents de sécurité).

\section{La difficulté de recruter et d'employer des enseignants en nombre et qualité suffisants}

Néanmoins, le processus de formation, de recrutement, d'emploi et de promotion des enseignants pose encore de nombreux problèmes.

En premier lieu, la formation, l'emploi et la promotion des enseignants ne sont pas bien planifiés. L'augmentation rapide du nombre d'établissements scolaires exige un plus grand nombre d'enseignants que les établissements de formation des enseignants ne peuvent en produire. Ainsi, de nombreux enseignants du secondaire inférieur sont promus enseignants au niveau du secondaire supérieur après avoir reçu une formation courte et des enseignants du primaire deviennent enseignants du secondaire inférieur. Le besoin en enseignants dans les écoles primaires est alors comblé par des étudiants diplômés d'autres universités, recrutés d'abord comme enseignants « journaliers » puis enseignants titulaires après une année d'expérience de l'enseignement et une formation courte. De même, de nombreux enseignants promus directeurs d'établissement du fait de l'augmentation du nombre d'établissements scolaires n'ont pas reçu de formation adéquate en matière de direction et de gestion éducative.

17 En second lieu, bien que le gouvernement ait, dans une certaine mesure, augmenté le salaire des enseignants (170000 kyats en moyenne $\left.{ }^{2}\right)$, celui-ci demeure encore insuffisant pour faire face aux frais de subsistance mensuels. Enfin, le processus de recrutement, de formation et d'emploi des enseignants devrait davantage prendre en compte les besoins régionaux et l'équité sociale. Les minorités ethniques et les groupes socialement défavorisés, disposant de moins de capital que les Birmans et la population urbaine, ne sont pas recrutés de manière représentative. De ce fait, les enseignants birmans ou issus des zones urbaines peuvent rencontrer des difficultés dans les régions des minorités ethniques et les zones rurales éloignées. De tels facteurs peuvent aggraver le taux d'épuisement professionnel des enseignants comme les inégalités éducatives et de plus intensifier les conflits sociopolitiques existants, les minorités ethniques percevant l'absence d'équité et de représentativité dans les services publics (cf. South et Lall, 2016).

\section{Mieux articuler éducation formelle et non formelle}

Le développement et l'amélioration de l'éducation non formelle et l'articulation entre éducation formelle et non formelle figurent également parmi les principaux objectifs de la loi sur l'éducation et du Plan stratégique national. Celui-ci se fixe pour objectif d'atteindre environ 2,7 millions d'enfants (âgés de 5 à 16 ans) n'ayant jamais été inscrits dans des établissements scolaires publics et 3,5 millions d'analphabètes parmi les jeunes (âgés de plus de 15 ans) grâce à la promotion de l'éducation primaire et de l'enseignement secondaire inférieur non formels et à des campagnes d'alphabétisation. Le Plan stratégique national envisage donc de mettre en place un système national en faveur de l'éducation alternative. Il prévoit également la mise en place d'un système de certification pour les élèves issus du secteur non formel ainsi que la création de 
passerelles vers des apprentissages futurs, dans l'enseignement technique et professionnel ou d'autres formations professionnelles.

Une autre piste d'amélioration significative consisterait à autoriser des fondations éducatives issues de la société civile (par exemple la Thabyay Education Foundation), opérant en dehors du pays, à dispenser des formations pédagogiques et professionnelles aux groupes défavorisés du pays. Mais la loi sur l'éducation a restreint le champ de l'éducation non formelle aux enseignements extrascolaires se basant sur le curriculum national. Les écoles ethniques ne suivant vraisemblablement pas intégralement le curriculum national, la création de passerelles permettant de rapprocher ces établissements des établissements publics et de l'enseignement supérieur semble essentielle pour la consolidation durable de la paix et l'avenir du pays en tant qu'union fédérale (South et Lall, 2016).

\section{Le développement de l'enseignement technique et professionnel}

Le développement de l'enseignement et de la formation techniques et professionnels (EFTP) est considéré comme un élément essentiel pour la production de travailleurs qualifiés et le développement économique du pays. Les plans en faveur de l'EFTP prévoient notamment de favoriser la collaboration entre les différents prestataires, d'améliorer la qualité de la formation, de mettre en place des normes nationales et de cibler les personnes issues des groupes défavorisés. Il existe environ 372 centres d'EFTP gérés par différents ministères et prestataires privés. Le département de l'EFTP gère 7 instituts de formation professionnelle, 36 lycées technologiques, 3 écoles techniques supérieures et 22 instituts technologiques (TVET, n.d.). Trois instituts de formation professionnelle ont été ouverts ou fonctionnent grâce à la coopération de Singapour, du Japon ou de l'Allemagne. Les stagiaires de l'EFTP reçoivent 30000 kyats par mois et le gouvernement fournit le matériel pédagogique nécessaire aux cours depuis 2015. Le gouvernement recrute également davantage de tuteurs/formateurs pour l'EFTP et développe les diplômes et les formations courtes.

\section{La réforme de l'enseignement supérieur}

21 Les principaux objectifs de la réforme de l'enseignement supérieur concernent l'autonomie, la qualité, le système d'admission et la recherche. Les universités peuvent signer des protocoles d'accord avec des universités étrangères et gérer leurs budgets. Le département de l'enseignement supérieur envisage d'adopter un classement des universités, basé sur les publications. Un maître de conférences doit disposer de publications ou d'un diplôme de doctorat. Néanmoins, étant donné la profusion actuelle de revues factices et de recherches de faible qualité, le comité de l'enseignement supérieur formé par le Plan stratégique national devra sans doute préciser la réglementation.

Depuis 2018, onze universités ont adopté leurs propres systèmes d'admission. Les résultats obtenus à l'examen de fin d'études secondaires demeurent un critère crucial d'accès à l'enseignement supérieur, bien que ces universités affirment prendre en compte également les entretiens dans leur système d'admission. 


\section{Éducation et territoires} peuvent gérer à la fois les budgets de fonctionnement et les budgets d'investissement. Depuis 2013-2014, les établissements peuvent décider de quelle manière utiliser les subventions et les États et les régions peuvent recruter des enseignants de primaire «journaliers» et agir sur le déploiement des enseignants. Les objectifs du Plan stratégique national sont de renforcer la gestion structurelle du système, d'améliorer la collaboration entre les différents prestataires d'enseignement, de renforcer les capacités du personnel enseignant et de développer un système de gestion basé sur l'information et la technologie. L'ensemble des bureaux de l'éducation, au niveau des États et des régions, ont lancé leurs sites web et leurs pages sur les réseaux sociaux, tels que Facebook, afin d'améliorer la communication et la diffusion des politiques publiques, et de rendre les projets de loi accessibles au public.

\section{Défis d'avenir}

Depuis 2011, la Birmanie a introduit des changements positifs d'importance évoqués dans cet article. Néanmoins, de nombreux défis cruciaux demeurent pour parvenir à une consolidation durable de la paix et atteindre l'objectif national fixé. Si le programme de l'éducation de base a bien été révisé et l'organisation scolaire commence à évoluer, le curriculum favorise toujours la birmanisation de l'enseignement et le nombre total d'heures de scolarité demeure inchangé - 5 h30 par jour en moyenne, de juin à mars. En outre, sur les 5139305 élèves du primaire de l'année scolaire 2016-2017, seuls 840918 (16,36\%) achèveront probablement l'enseignement secondaire. En dépit des millions dépensés et des efforts considérables consentis, le Plan stratégique national ne parvient à proposer des parcours éducatifs qu'à un quart de la population actuelle des élèves du primaire et, au mieux, à terme, à 30 \% d'entre eux.

Aussi, afin que l'éducation puisse être une force centrale pour la réalisation de l'objectif national 2030 de paix durable et de transformation sociale du pays, de plus amples efforts devront être consentis afin de proposer des parcours scolaires pour la majorité d'enfants non scolarisés, en allant au-delà du Plan stratégique national.

\section{BIBLIOGRAPHIE}

CSO (2017) : 2017 Myanmar Statistical Yearbook, Nay Pyi Taw : Ministry of Planning and Finance.

JOLLIFFE K. (2014) : Ethnic conflict and social services in Myanmar's contested regions, The Asia Foundation.

MOBA (2019): The number of students at youth training schools in 2017-2018 AY, janvier 2019, [en ligne] [https://bit.ly/2ScGlfc]

Revue internationale d'éducation de Sèvres, 80 | avril 2019 
NESP (2016) : National Education Strategic Plan 2016-2021, Nay Pyi Taw : Ministry of Education.

SOUTH A. et LALL M. (2016) : Schooling and conflict : Ethnic education and mother tongue-based teaching in Myanmar, USAID/ The Asia Foundation. TVET (n.d.) : Training schools : Technical and Vocational Education and Training. [en ligne] [http://www.tvetmyanmar.gov.mm/en/trainingschools]

\section{NOTES}

1. Environ $18 €$. (NdlR)

2. Autour de $100 €$. (NdlR)

\section{INDEX}

Mots-clés : système éducatif, politique éducative, curriculum, enseignant Index géographique : Birmanie

Keywords : educational system, educational policy, curriculum, teachers

Palabras claves : sistema educativo, política educacional, curriculum, docente

\section{AUTEURS}

\section{KAM TUNG TUANG SUANTE}

Kam Tung Tuang (Peter) Suante est né dans le village de Tuisau, dans l'État de Chin, au Myanmar (Birmanie). Après avoir obtenu une licence à l'Université d'éducation de Sagaing, il a travaillé en tant qu'enseignant du secondaire à Naga Hills, l'une des régions les plus défavorisées du pays. Il a ensuite étudié la sociologie de l'éducation à l'Université d'éducation de Sagaing puis le leadership et le management en éducation (master) à l'Université normale de Beijing. Il est actuellement doctorant à l'Université de Hong Kong. Ses recherches portent sur les politiques éducatives et le soutien privé. Courriel : suantepeter@gmail.com 